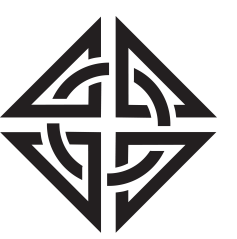

Sharif University of Technology

Scientia Iranica

Transactions A: Civil Engineering

http://scientiairanica.sharif.edu

\title{
The interaction between activity choice and duration: Application of copula-based and nested-logit models
}

\author{
F. Jafari Shahdani, A. Rasaizadi, and S. Seyedabrishami* \\ Department of Civil and Environmental Engineering, Tarbiat Modares University, Tehran, P.O. Box 14115-111, Iran.
}

Received 16 February 2020; received in revised form 27 June 2020; accepted 3 October 2020

\author{
KEYWORDS \\ Non-mandatory \\ activities; \\ Joint model; \\ Activity choice; \\ Activity duration; \\ Copula; \\ Nested-logit.
}

\begin{abstract}
There is a relationship between choosing an activity and its duration, especially for non-mandatory activities. A number of studies have analyzed the decisions about an activity type and its duration independently, while some others have used joint models. This paper contributes to the body of knowledge using nested-logit and copula-based models for assessing the existence of interdependency between, or a hierarchy of, the choice of non-mandatory activity and its relative duration. In the case of the nestedlogit model, it is assumed that error terms of these decisions are interrelated, although one is influenced by another. In contrast, the copula-based model can facilitate making a spatial error correlation between observational units without imposing the assumption of restrictive distribution on the dependency structures between the error components. The data available from Qazvin, a city in Iran, were used for estimating both nested-logit and copula-based models and the best variables explaining both choices for each model were selected. The final models were compared in terms of log-likelihood at convergence and adjusted likelihood ratio index. The results indicated that there were some common influential observed and unobserved factors between these decisions. Also, the copula-based joint model with $\rho_{0}^{2}$ equal to 0.134 outperformed nested-logit models and provided better explanatory power.

(C) 2021 Sharif University of Technology. All rights reserved.
\end{abstract}

\section{Introduction}

Transportation planning is significantly affected by travelers' decision-making. The type of activity and its duration represent two major decisions required for planning. Individuals' choice of activity has been previously investigated to study travel behavior and other different aspects of activity choice such as type, timing, and duration have been examined separately at the outset; however, thanks to recent advances in recent

\footnotetext{
*. Corresponding author. Tel.: +982182884964

Fax: +9821 82884614

E-mail addresses: F_jafari@modares.ac.ir (F. Jafari

Shahdani); arash_rasaizadi@modares.ac.ir (A. Rasaizadi);

seyedabrishami@modares.ac.ir (S. Seyedabrishami)
}

research, these elements have been highlighted simultaneously, shedding light on their significance $[1,2]$.

Identification of more realistic modeling and the factors influencing variations in the timing of activities could result in more precise planning and scheduling for behavioral models. Moreover, such identification would be helpful for authorities to implement effective strategies to reduce network congestion, especially during peak hours. Many policies require information on the timing behavior of activities, e.g., telecommuting, flexible work hours, and dynamic congestion pricing $[3,4]$.

Copious research in the field of activity type choice and duration highlights the significance of examining these two decisions [5-7]. Given the close relationship between and the direct effect of these two decisions on each other, the necessity to examine this 
interaction is demonstrated. This interaction can be tested in the form of either a nested-logit model or the copula-based joint models. Although the interaction between these two decisions is investigated through nested-logit models, this relationship is hierarchical and the simultaneous model cannot be called. In other words, high and low levels of the nested-logit model indicate the sequence between two decisions. It appears that the nested-logit model has not yet been used in terms of activity choice and duration. Although some researchers have considered this model a joint one in the form of cross-nested-logit model (e.g., [8,9]), many others have used that as a single choice model in case of different choices including mode choice and destination choice $[10,11]$.

In the context of copula-based models, there is no such sequence between two choices, i.e., both decisions are paid at the same level. Since the purpose of this study is to examine the relationship between two decisions of activity type and duration, both structures are examined and the results are compared subsequently. The existence of the nested-logit model at the mentioned levels with different decisions as well as the copula-based model and the evaluation of their results could determine the possibility of examining the simultaneity or existence of a hierarchy as well as the position of each decision in a hierarchical structure.

Since the type of activity has a strong effect on the activity duration decision, it is important to estimate a unique model for each type of activity. Therefore, this study concentrated on non-mandatory travels because of their nonroutine and flexible nature. These activities were categorized into four groups:

1. Shopping including arbitrary shopping activities;

2. Recreation including optional leisure or pilgrimage activities;

3. Accompanying including picking-up/dropping-off somebody from somewhere;

4. Personal activities including optional visits to the doctor, office, close relatives, and other optional activities.

This research aims to contribute to the existing literature on travel behavior using a copula-based model as a novel approach to assessing the existence of interdependency between the choice of non-mandatory activities and their relative duration using the data obtained from the travel survey of Qazvin, a city in Iran. Since this model has been calibrated for local data in a developing country for the first time, this paper aims to assess the interdependency between individuals' activity choice and duration, compared with a set of nested-logit models. For a better understanding of human decision-making, factors with a significant effect on trip aim or activity duration, or both of them, and the interpretation of these factors are investigated. It should be noted that since some people initially decide about the trip mode in case of non-mandatory trips and then, decide about the aim of the trip, this paper assumes that the choice of activity and duration are determined only after the mode choice. Born et al. [12] implied in their research that activity durations for automobile users were directly related to vehicle soak time. Finally, the research aims can be summarized as follows:

- Capturing the interdependency between the choice of non-mandatory activities and their duration;

- Using local data as a developing country;

- Finding effective independent variables in traveler's decision-making;

- Employing the copula-based model as a joint model and nested logit model as a hierarchy model and comparing their performances;

- Interpreting estimated coefficients and their significances.

The remainder of this paper is developed as follows. Section 2 provides a brief review of the existing literature on activity timing and duration choice. Section 3 describes the framework for the copula-based joint model and the nested-logit model with a discussion of the data used in the paper. After presenting the results of the models, description and comparison of the results are presented. In the final section, conclusions and some directions for future research are put forward.

\section{Previous studies}

Activity participation and time in the transportation field use have received significant attention. According to the activity-based travel theory, trips could be considered as a necessary means of connecting distinct activities that logically precede or accompany these activities [13]. This means that the scheduling of trips relies on not only time-of-day travel characteristics (such as travel time and delay) but also the preferences regarding the timing and duration of activities (e.g., [14]). Recently, studies have investigated the effect of social networks on different characteristics of non-mandatory activity participation [15-17]. While such studies provide a better understanding of the determinants of participation in non-mandatory activities, they generally require specialized data collection efforts. Typical travel data sets do not contain such detailed information, making it difficult to use the proposed frameworks to study non-mandatory participation decisions of special events [12].

On the other hand, the importance of modeling activity duration has been investigated quite well in the 
literature. For modeling the duration of activities, various approaches have been proposed. Several authors (e.g., [18,19]) have proposed hazard-based duration models. In hazard models, the duration is taken as a continuous variable and the probability of an activity ending is described depending on the time spent on that activity. A series of socio-economic variables typically affect the hazard rate. However, hazard modeling provides a statistical approach to modeling distributions of durations without any underlying behavioral theory. In response to this flaw, various approaches use a utility-based framework to elaborate the scheduling of activities in the course of continuous time. The central idea behind using these methods is that there is a marginal utility (which can differ over time) for each time of the day, representing the utility derived from one time unit of activity participation. The idea of utility rate was first implemented in the context of activity scheduling by Polak et al. [20] and was further refined by Ettema and Timmermans [14] who proposed specific functional forms for the functions of the utility rate. However, the variation of travel time during the day was ignored in this approach. Bhat and Steed [21] used a nonparametric hazard model for the time allocation of shopping activities, and Habib et al. [22] and Habib [23] presented parametric hazard models for continuous time departures. Furthermore, Leszczyc and Timmermans [24] estimated activity durations using hazard models and then, employed Markov transitions to predict the choice and duration of the next activity based on the duration and type of the previous activity. Chen and McKnight [25] discussed the impact of built environment features on homemakers' time use behavior. The impact on allocation time of homemakers to out-of-home discretionary activities was visible.

In another stream of research, Kapur and Bhat [26] investigated the participation of people in various activities on weekends. The results indicated that low-income households tended to be more active at home on weekends, pointing to a financial constraint of more costly out-of-home activities. Besides, sociodemographic characteristics might affect the choice of activities, too. For example, women and married people are more likely to have indoor activities than men and singles, respectively, while singles and middleaged people (aged 40-60) are more interested in outof-home activities. $\mathrm{Xu}$ et al. [27] presented a specific type of Household Activity Pattern Problem (HAPP) that could predict not only the activity chains but also the activity choice and the time allocated to activities via multinomial logit method, while the choice set in this paper is based on the data disregarding sociodemographic features and personal characteristics.

Frei et al. [28] presented a joint model of travel time activity participation and service value that was modeled as a latent variable. Although they identified the parameters involved in the mentioned participation in a specific type of activity (passive leisure, active leisure, and work), they failed to investigate duration as a factor.

There is another stream of research that has treated activity type and duration choices as simultaneous choices. In the context of daily activity scheduling, Bhat et al. analyzed activity choice and duration in several studies (e.g., $[5,26,29]$ ) and could illustrate the importance of socio-demographic variables, vehicle ownership, accessibility to public transport infrastructure, and land use characteristics in the choice of various activities. The mentioned authors used the Multiple Discrete Continuous (MDC) framework for analyzing the activity duration choices, while they did not make any comparison between their results and researches that assumed the activity type and duration choices as sequential choices.

Finally, a recent contribution by Pawlak et al. [30] established a flexible link among a discrete choice model of the selection of activity type, a hazard-based model for the duration of each activity, and a log-linear model of productivity using copulas in the context of rail travels. While they demonstrated links among participation in a particular type of activity, duration, and productivity aspects, they did not explore the interdependency between activity type and duration choice. The existence of some commonly observed and unobserved factors involved in making these two choices indicates that they do not function in isolation; no study has specified the simultaneity or hierarchy of these decisions. Moreover, joint modeling using a copula-based theory is used to a greater degree for other transportation modeling cases. For instance, Ermagun et al. [31] pointed to the superiority of a copula-based model for modeling mode choice and escort decisions for school trips. In another research, Rasaizadi and Kermanshah [32] explored the interrelation between mode and number of stops using the copula-based model.

Overall, although the models of time use have been improved over recent decades, to date, no research has been done on assessing the existence of interdependency between or hierarchy of type and duration of non-mandatory activity choices. This paper tries to address this gap by processing the selection of non-mandatory activity type and duration choices by comparing the results of a set of nested-logit models with a copula-based model for the Iranian community using the origin-destination data of Qazvin.

\section{Methods}

In this section, the proposed models are introduced. The choice set in trip objectives included shopping, personal, recreational, and accompanying purposes and 
the duration allocated to the type of discretionary activity was categorized as low, medium, and long durations in this study. At first, the joint multinomialordered logit model using copula function was introduced and then, the nested-logit model was presented as a Generalized Extreme Value (GEV) model.

\subsection{Copula-based joint model}

This study employed copula-based joint modeling to capture the reciprocal impact between activity choice and activity duration for non-work trips. This joint model estimates two discrete choice models, that is, the multinomial logit model for activity choice and ordered logit model for activity duration choice. Ultimately, all the coefficients and parameters of the two models are estimated simultaneously in an integrated model. Each model is first described independently.

The multinomial logit model is used for modeling activity choice. Let $q$ and $i$ be the indices for individual and activity, respectively. Also, let $h_{q j}$ (Eq. (1)) be the latent utility for the activity selected by individual $q$ :

$$
h_{q i}=\beta x_{q i}+\varepsilon_{q i},
$$

where $x_{q i}$ is the column vector of independent variables specified to mode $i$ and $\beta$ is the vector of the parameters to be estimated. $\varepsilon_{q i}$ represents an idiosyncratic error term that is distributed identically which is independently characterized by extreme value. Individual $q$ chooses activity $i$ if (Eq. (2)):

$$
h_{q i}>\max _{j=i} h_{q i}
$$

Define Eq. (3):

$$
v_{q i}=\left\{\max _{j \neq i} h_{q j}\right\}-\varepsilon_{q i} .
$$

$v_{q i}$ follows the logistic distribution.

Define $r_{q i}, r_{q i}=1$, if the $i$ th activity is chosen by the $q$ th individual; otherwise, $r_{q i}=0$. The $i$ th activity is chosen by the $q$ th individual if (Eq. (4)):

$$
\beta x_{q i}>v_{q i}
$$

The implied marginal distribution of $v_{q i}$ is considered as the probability of choosing activity $i$ by individual q. It is obtained through Eq. (5):

$$
F_{i}\left(\beta x_{q i}\right)=\operatorname{Pr}\left(v_{q i}<\beta X_{q i}\right)=\frac{\exp \left(\beta X_{q i}\right)}{\sum_{j} \exp \left(\beta X_{q j}\right)} .
$$

The ordered logit model is used for modeling activity duration. Let $s_{q i}^{*}$ be the propensity of having a longer activity duration of the $q$ th individual that chooses activity $i . s_{q i}$ represents the activity duration levels: low, medium, and high. $s_{q i}$ is observed only for the chosen activity $i$. In the ordered logit structure, define Eq. (6):

$$
s_{q i}^{*}=\gamma z_{q i}+\eta_{q i}, \quad s_{q i}=k, \quad \text { if } \quad \delta_{i, k-1}<s_{q i}^{*},
$$

where $z_{q i}$ is the column vector of the independent variables specified to mode $i$ and $\gamma$ is the vector of coefficients to be estimated. $\eta_{q i}$ represents an idiosyncratic error term assumed to be standard logistic distributed with the marginal cumulative distribution function given $\mathrm{G}($.$) . The \delta$ terms are the threshold bounds between levels of activity and provide a relation between the propensity of having more activity duration and the observed activity duration. By convention, $\delta_{i, 0}=-\infty$ and $\delta_{i, K}=+\infty$ for each mode $i$, where $k$ is an index for activity duration ( $k=1$ equals to low, $k=2$ equals to medium, and $k=3$ equals to high).

The probability that the individual $q$ chooses activity $i$ and activity duration $k$ can be written as in Eqs. (7) and (8):

$$
\begin{aligned}
\operatorname{Pr}\left[r_{q i}=\right. & \left.1, s_{q i}=k\right]=\operatorname{Pr}\left[v_{q i}<\beta x_{q i}, \delta_{i, k-1}-\gamma z_{q i}\right. \\
& \left.<\eta_{q i}<\delta_{i, k}-\gamma z_{q i}\right], \\
\operatorname{Pr}\left[r_{q i}=\right. & \left.1, s_{q i}=k\right]=\operatorname{Pr}\left[v_{q i}<\beta x_{q i}, \eta_{q i}<\delta_{i, k}-\gamma z_{q i}\right] \\
& -\operatorname{Pr}\left[v_{q i}<\beta x_{q i}, \eta_{q i}<\delta_{i, k-1}-\gamma z_{q i}\right] .
\end{aligned}
$$

In Eq. (8), the bivariate cumulative distribution function between the error terms of the two models is required and it is provided by copulas. In 1959, in response to a query of M. Fréchet, A. Sklar introduced copulas. A copula is a multivariate distribution function over the unit cube that generates a stochastic dependence relationship among random variables and pre-specified marginal distributions. In the bivariate case, let $C$ be a two-dimensional copula of uniformly distributed random variables $U_{1}$ and $U_{2}$ with the support contained in [0,1] [33]. Then $C\left(u_{1}, u_{2} ; \theta\right)=$ $\operatorname{Pr}\left[U_{1} \leq u_{1}, U_{2} \leq u_{2}\right]$ [34], where $\theta$ is a parameter of the copula commonly referred to as the dependence parameter. A copula allows the generation of joint bivariate distribution functions with given marginals. A rich set of bivariate copulas is available to generate the dependence between the random variables including the Gaussian copula, the Farlie-Gumbel-Morgenstern (FGM) copula, and the Archimedean class of copulas (including Clayton, Gumbel, and Frank). The reader is referred to Bhat and Eluru [33] for more details of the copulas.

Also, a m-copula can be defined as $m$-dimensional Cumulative Distribution Function (CDF) whose support is contained at $[0,1]$ and whose one-dimensional marginal is uniform at $[0,1](\mathrm{Eq} .(9))$ : 


$$
\begin{aligned}
F\left(x_{1}, \ldots, x_{m}\right) & =F\left(F_{1}^{-1}\left(u_{1}\right), \ldots, F_{m}^{-1}\left(u_{m}\right)\right) \\
& =\operatorname{Pr}\left[U_{1} \leq u_{1}, \ldots, U_{m} \leq u_{m}\right] \\
& =C\left(u_{1}, \ldots, u_{m} ; \theta\right),
\end{aligned}
$$

where $\theta$ is called the dependence parameter, which measures the dependence between marginals. Therefore, Eq. (8) is rewritten as in Eq. (10):

$$
\begin{gathered}
\operatorname{Pr}\left[r_{q i}=1, s_{q i}=k\right]=C_{\theta}\left(u_{q i 1}, u_{q i, k, 2}\right) \\
-C_{\theta}\left(u_{q i 1}, u_{q i, k-1,2}\right) .
\end{gathered}
$$

Copula is applicable to many research scopes. For example, Liu et al. [35] constructed the correlation and its corresponding risk measurement models based on the mixed Clayton copula. Pan et al. [36] developed a copula-Bayesian approach to modelling risks associated with dependent systems which is reliable based on model validation. Also, Seyedabrishami et al. [37] investigated a joint model for a number of cars and fuel types using copula functions.

In Eq. (9), parameter $(\theta)$ shows the correlation between $v_{q i}$ and $\eta_{q i}$. Of note, the correlation between $\varepsilon_{q i}$ and $\eta_{q i}$ has a reverse sign.

In this paper, two types of copula are used: Product and Frank copulas. In product copula, copula dependence parameters are equal to zero. Thus, it is used to depict the independency between error terms; however, Frank copula, a well-known copula function, considers the correlation between marginals. Table 1 displays the characteristics of these copulas.

To estimate the joint multinomial-ordered model, let $1[$.$] be an indicator function taking the value of$ unity if the expression in parenthesis is true and 0 otherwise. Define Eq. (11):

$$
M_{q i k}=1\left[r_{q i}=1\right] * 1\left[s_{q i}=k\right] .
$$

Finally, the log-likelihood function is as Eq. (12):

$$
\log L=\left(\sum_{q=1}^{Q}\left(\sum_{i=1}^{I} \sum_{k=1}^{K} M_{q i k} \log \left[\operatorname{Pr}\left(r_{q i}=1, s_{q i}=k\right)\right]\right)\right.
$$

By maximizing this function, all the coefficients and parameters will be estimated. This maximation is accomplished using the $\mathrm{R}$ programming language (https://www.r-project.org/about.html and https://www.rstudio.com/).

\subsection{Nested-logit model structure}

The nested-logit model is one of the most practical GEV models. In the structure of the nested-logit model, interdependent alternatives are placed in similar nests. The nested-logit model is designed on the basis of the random utility theory similar to the multinomial logit model, although their replacement patterns are different. This model can solve a part of the independence between irrelevant alternatives that are ignored in the multinomial logit model. The assumption of independence of irrelevant alternatives is established in one nest, but this assumption does not exist in different nests. Utility function in the nestedlogit model is defined as Eq. (13):

$$
U_{n j}=W_{n k}+Y_{n i}+\varepsilon_{n j},
$$

where $W_{n k}$ is a part of the utility function for person $n$, is dependent on variables describing nest $k$, and is the same for all of the nest alternatives. $Y_{n i}$ is a part of the utility function for person $n$, is dependent on variables describing alternative $i$, and changes for each alternative in the nest and $\varepsilon_{n j}$ is a random variable whole value is not observed by the researcher. If $B_{k}$ is a high-level alternative and $i \in B_{k}$ and $i$ is a low-level alternative in the structure of the nested-logit model, the probability of choosing the alternative $i$ will be obtained through Eq. (14) [38]:

$$
P_{n i}=P_{n i \mid B_{k}} P_{n B_{k}}
$$

In particular, the marginal and conditional probabilities can be expressed as Eqs. (15) and (16):

$$
\begin{gathered}
P_{n B_{k}}=\frac{e^{W_{n k}+\lambda_{k} I_{n k}}}{\sum_{l=1}^{k} e^{W_{n l}+\lambda_{l} I_{n l}}}, \\
P_{n i \mid B_{k}}=\frac{e^{Y_{n i} / \lambda_{k}}}{\sum_{j \in B_{k}} e^{Y_{n i} / \lambda_{k}}},
\end{gathered}
$$

where:

$$
I_{n k}=\ln \sum_{j \in B_{k}} e^{Y_{n i} / \lambda_{k}}
$$

where $i$ denotes the alternative $i$ at a low level and $B_{k}$ denotes nest $k$ at a high level. $P\left(n i \mid B_{k}\right)$ is the probability of choosing $i$ at a low level under the

Table 1. Characteristics of frank and product copulas.

\begin{tabular}{lcc}
\hline Copula & $\boldsymbol{C}\left(\boldsymbol{u}_{\mathbf{1}}, \boldsymbol{u}_{2}\right)$ & Dependence parameter range \\
\hline Product & $u_{1} u_{2}$ & - \\
Frank & $-\theta^{-1} \log \left\{1+\frac{\left(e^{-\theta u_{1}}-1\right)\left(e^{-\theta u_{2}}-1\right)}{e^{-\theta}-1}\right\}$ & $(-\infty, \infty)$ \\
\hline
\end{tabular}


condition of choosing $B_{k}$ nest at a high level. $P\left(n B_{k}\right)$ is the probability of choosing $B_{k}$ alternative at a high level for observation. $P(n i)$ is the probability of choosing an alternative at a low level for observation $n$. $\quad \lambda_{k}$ is the scale parameter for nest $k$. The maximum likelihood estimation is used for modeling discrete choice models and defining the alternative utility function. Calculation of likelihood function and the probability of selecting alternatives are written as in Eqs. (18) and (19):

$$
\begin{aligned}
& L(\beta)=\prod_{n=1}^{N} \prod_{i}\left(P_{n i}\right)^{y_{n i}}, \\
& L L(\beta)=\sum_{n=1}^{N} \sum_{i} y_{n i} \ln P_{n i},
\end{aligned}
$$

where $\beta$ is a vector including utility function variables. $N$ is the number of observations in a given sample. $p_{n i}$ denotes the probability of choosing the alternative $i$ if $i$ is a member of feasible alternatives set for the person $n$, and $y_{n i}$ is one if the alternative $i$ is selected by the person $n$ and is zero otherwise. In order to maximize $L$, its distribution derivations $L L(\beta)$ should be equal to zero for each of the coefficients of the utility function and the values of the utility function parameters are obtained by solving the simultaneous equations.

\section{Data}

The data used in this research were collected from a questionnaire designed in 2012 through a comprehensive transportation study of Qazvin-Iran [39], which examined the travel information of 35420 individuals on a weekday including the purpose of the travel, the mode of travel, the start and end time of the travel, and the time allocated to each activity. Moreover, in this survey, socio-economic information has been gathered in individual and household dimensions. This research has only focused on individuals who have done at least one discretionary activity during the day. In fact, these types of trips have been investigated because individuals have greater freedom in choosing trips with non-mandatory purposes and also the time allocated to each activity is more flexible. After further processing for cleaning data with missing information on important variables (e.g., socio-economic variables and travel information), the sample population used in this paper includes 4290 individuals who have made trips with non-mandatory purposes (except work, education, and return home). Qazvin-related data as a particular case study is selected for two reasons; first, there is no similar study on this topic for such a community with a focus on developing countries. Second, this dataset is available for Qazvin Iran, which is a large metropolitan area in Iran with similarities to other major cities in the country. Figure 1 shows the GIS map of Qazvin.

Out of the information collected and reshaped into a database, with regard to previous literature, those variables that have been effective in determining

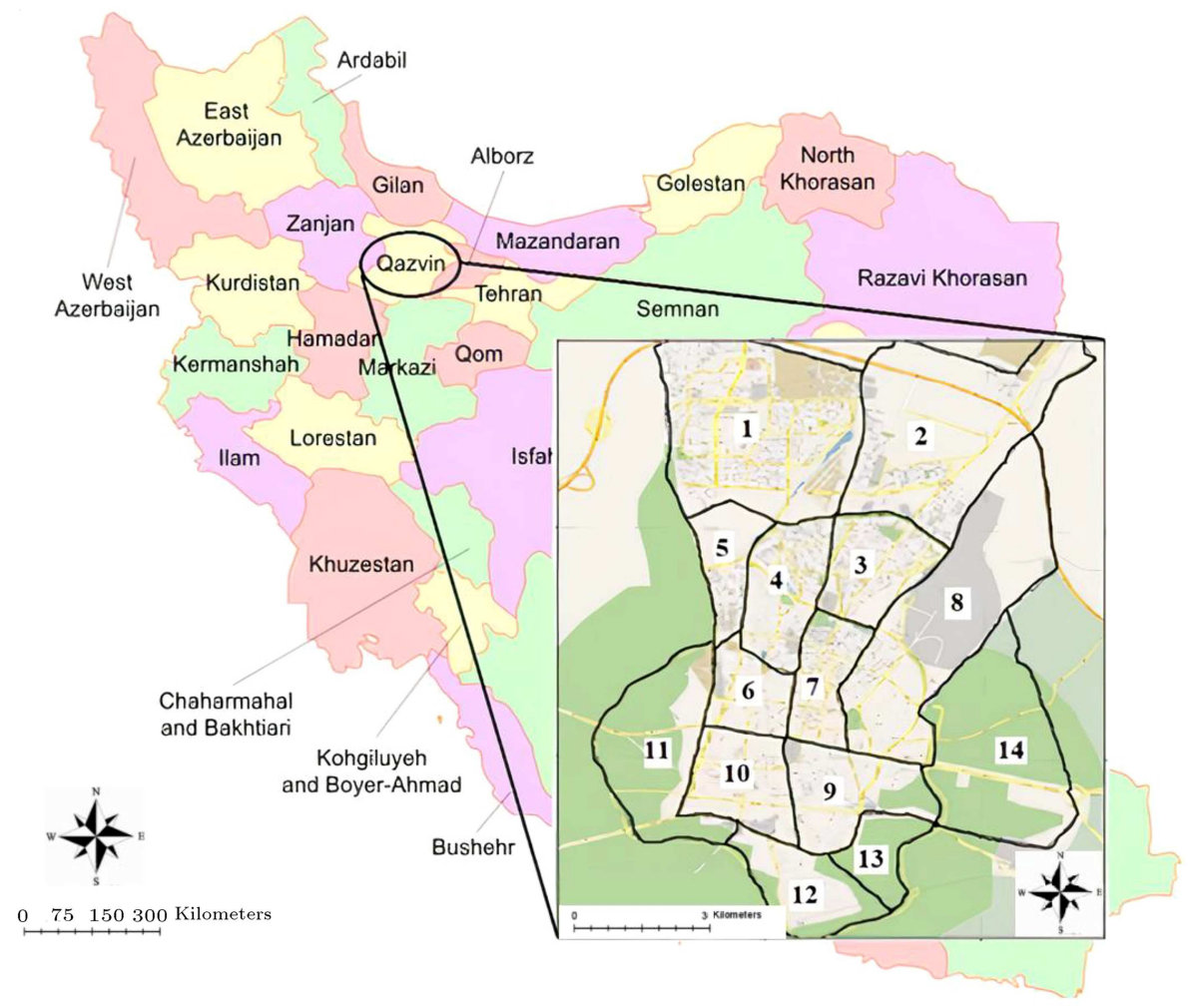

Figure 1. Location of Qazvin in Iran and its traffic analysis zones [40]. 
the choice and duration of activities were identified and classified in Table 2. In order to calibrate the models of choosing the type and duration of activity, with respect to the similarity existing between various alternatives and considering the proportion of frequency of observations related to them, nonmandatory activities were divided into four groups: 1) shopping (including arbitrary shopping activities), 2) recreational (including optional leisure or pilgrimage activities), 3) accompanying (including accompanying someone along), and 4) personal activities (including optional visits to the doctor, office, close relatives and other optional activities). Given the weekday information and the fact that people often engage in compulsory activities about two-thirds of the day, three types of duration were considered as the times allocated to each non-mandatory activity: short duration (less than an hour), medium duration (one to two hours), and long duration (more than two hours). The frequency of the categories is displayed in Tables 3 and 4 .

\section{Results}

At first, let us consider nested-logit with the activity choice at the upper level and activity duration as 'model A' and another nested-logit model as 'model B'. The results of each model are interpreted and will be presented in this section. Initially, the results of the copula-based model are displayed in Table 5 . Thereafter, Tables 6 and 7 present estimation results for models A and B, respectively.

\section{Discussion}

\subsection{Copula dependence parameters}

Copula dependence parameters show the correlation between two random variables, $v_{q i}$ and $\eta_{q i}$ [33]. The correlation between $\varepsilon_{q i}$ and $\eta_{q i}$, considered as random terms for the two models, exhibited reverse sign. So, there are some observed and unobserved factors that affect these decision-making cases simultaneously. Unobserved factors increase the probability of choosing shopping and accompanying activities and at the same time, increasing the probability of selecting trips with a long activity duration. Furthermore, the unobserved factors have a different effect on the probability of choosing the two other activities. That is, if these factors increase (or decrease) the probability of choosing personal and recreational activities, they have a reverse effect on the probability of choosing activities with a long duration, synchronically.

\subsection{Interpretation of the estimated coefficients in the copula-based model}

Regarding estimated coefficients, shopping activity is the least desired activity for individuals aged between 5 and 30. Personal activities are attractive for individuals over 31 years old (definitions of attraction in this paper are the same as those in other previous papers in this field) [41]. Recreational and accompanying trips have the least and the most desired ones for individuals aged between 31-41. The probability of choosing long activity duration decreases for the individuals between 5-18 years and increases for the individuals between 19-31 years old.

Moreover, results indicate that men prefer to have personal and recreational trips along with a long activity duration in comparison with women. Individuals with education-related and other jobs find shopping and recreational activities more attractive than those with administrative and service jobs. Individuals with administrative and service jobs, on the other hand, have a more tendency towards high activity duration.

Having a driving license increases the probability of choosing accompanying trips and decreases the probability of choosing personal and recreational trips and long-duration activities.

Considering the educational level, individuals with low or medium levels of education prefer to have shopping trips. Personal and accompanying trips seem not to be attractive for individuals with high levels of education as compared to other individuals. These individuals choose activities with a short duration due to their limited time-budget. Upon increasing the household size, the probability of choosing personal trips and short activity durations increases.

Shopping is not an attractive mode when individuals use a private car, a taxi, and a motorcycle, as compared to using a bus and opting for active modes. Individuals prefer to use a private vehicle in accompanying trips. Bus and active modes are attractive modes for personal and accompanying trips, respectively. For long activity duration trips, the bus has the highest probability of choosing, while active mode has the least probability.

\subsection{Interpretation of the estimated coefficients of model $A$}

The model parameters have the expected correct signs. Regarding estimated coefficients, those aged between 5 and 18 years old have more favorable recreational trips with a short duration than those with shopping, personal, and accompanying activities with all duration types. Shopping activities take less time for older people over 40 years of age. These people prefer not to take their personal trips in short and medium durations. In addition, accompanying activities with medium and long durations are more favorable for them than short-duration trips due to having more free time as a result of retirement. Men are more inclined to do short-duration shopping and personal trips than 
Table 2. Effective variables for the selection of non-mandatory activities and durations.

\begin{tabular}{|c|c|c|c|}
\hline Variable & Category & Description & Frequency \\
\hline \multirow{4}{*}{ Age } & Age $5-18$ & 1 if the individual is between 5 and 18 years old, 0 otherwise & $10 \%$ \\
\hline & Age $19-30$ & 1 if the individual is between 19 and 30 years old, 0 otherwise & $24 \%$ \\
\hline & Age $31-41$ & 1 if the individual is between 31 and 41 years old, 0 otherwise & $31 \%$ \\
\hline & Age $>41$ & 1 if the individual is more than 41 years old, 0 otherwise & $35 \%$ \\
\hline Sex & Sex & 1 if the individual is male, 0 otherwise & $38 \%$ \\
\hline \multirow{6}{*}{ Job } & SERV job & 1 if the individual has a service job (driver, farmer, & $23 \%$ \\
\hline & & seller, manual worker and master of work), 0 otherwise & \\
\hline & ADM job & & $18 \%$ \\
\hline & & (employee, military, and cultural occupations), 0 otherwise & \\
\hline & EDU job & $\begin{array}{l}1 \text { if the individual has an educational job } \\
\text { (students and college students), } 0 \text { otherwise }\end{array}$ & $24 \%$ \\
\hline & RE-HM job & $\begin{array}{l}1 \text { if the individual has a job except for above } \\
\text { (retired and homemaker), } 0 \text { otherwise }\end{array}$ & $35 \%$ \\
\hline Driver license & $\mathrm{DL}$ & 1 if the individual has a driver license, 0 otherwise & $49 \%$ \\
\hline \multirow{3}{*}{ Education } & Low EDU & 1 if the individual does not have a high school degree, 0 otherwise & $39 \%$ \\
\hline & Med EDU & 1 if the individual has a college degree, 0 otherwise & $38 \%$ \\
\hline & High EDU & 1 if the individual has a master of science degree and upper, 0 otherwise & $23 \%$ \\
\hline \multirow{5}{*}{ Mode } & Private & 1 if the individual makes the trip by private car, 0 otherwise & $25 \%$ \\
\hline & Taxi & 1 if the individual makes the trip by taxi, 0 otherwise & $30 \%$ \\
\hline & Bus & 1 if the individual makes the trip by bus, 0 otherwise & $15 \%$ \\
\hline & Motorcycle & 1 if the individual makes the trip by motorcycle, 0 otherwise & $13 \%$ \\
\hline & Active & 1 if the individual makes the trip by bicycle or walking, 0 otherwise & $17 \%$ \\
\hline $\begin{array}{l}\text { Household } \\
\text { size }\end{array}$ & HHSZ & Number of individuals in a household & $\begin{array}{l}\text { Average } \\
=4\end{array}$ \\
\hline $\begin{array}{c}\text { Car } \\
\text { ownership }\end{array}$ & $\mathrm{CO}$ & Number of vehicles per person in the household & $\begin{array}{l}\text { Average } \\
=0.8\end{array}$ \\
\hline $\begin{array}{l}\text { Bike } \\
\text { ownership }\end{array}$ & $\mathrm{BO}$ & Number of bicycles per person in the household & $\begin{array}{l}\text { Average } \\
=0.6\end{array}$ \\
\hline
\end{tabular}


Table 3. Frequency of the alternatives.

\begin{tabular}{llc}
\hline Model & Alternatives & Frequency \\
\hline \multirow{4}{*}{ Activity } & Shopping & $33 \%$ \\
& Personal & $25 \%$ \\
& Recreational & $22 \%$ \\
& Accompanying & $20 \%$ \\
& & \\
\multirow{2}{*}{ Duration } & Short duration & $35 \%$ \\
& Medium duration & $36 \%$ \\
& long duration & $29 \%$ \\
\hline
\end{tabular}

Table 4. Frequency of the joint alternatives.

\begin{tabular}{clc}
\hline \multicolumn{1}{c}{ Model } & Alternatives & Frequency \\
\hline \multirow{4}{*}{ Short duration } & Shopping & $13 \%$ \\
& Personal activities & $7 \%$ \\
& Recreational & $6 \%$ \\
& Accompanying & $8 \%$ \\
& & \\
Medium duration & Shopping & $12 \%$ \\
& Personal activities & $9 \%$ \\
& Recreational & $8 \%$ \\
& Accompanying & $5 \%$ \\
& & $8 \%$ \\
& Shopping & $9 \%$ \\
& Personal activities & $8 \%$ \\
& Recreational & \\
& Accompanying & \\
\hline \multirow{5}{*}{ Long duration } & & \\
& &
\end{tabular}

women. On the contrary, women devote more time to shopping and accompanying trips than men. Lowduration shopping is the most attractive one for those with administrative jobs and is the least desired for retired individuals and homemaker people. Also, longduration accompanying activities are the least favorite trips for those with educational jobs and the most desirable one for the retired and homemaker people. In general, homemaker women and retired people opt for a longer period of time for different activities with regard to having a longer free time. Having a driver's license increases the probability of choosing shopping and recreational activities with a long duration.

By investigating the obtained coefficients for education, it became clear that people with higher levels of education were more inclined toward short- duration activities. In contrast, less educated people chose longer duration activities. The most effective factor in these alternatives is the time constraints for people with higher education. The probability of choosing shopping and recreational activities with a long duration will be increased with increase in the household size. The more vehicle ownership per person in the household, the higher the probability of selecting higher duration shopping and recreational trips. Conversely, accompanying activities with a short duration for people would be more desirable. The likelihood of selecting short-duration recreational activities will be decreased with an increase in bicycle ownership. The coefficients obtained for the trip mode indicate that private car is an appropriate alternative to medium-duration personal trips and short-duration accompanying trips. In contrast, the probability of choosing this vehicle decreases for accompanying trips with a long duration. Bus is a vehicle that has less utility for short-duration activities, and individuals prefer to do long-duration activities by bus. The probability of choosing medium-duration personal activities increases while using a motorcycle as a vehicle. Short-duration shopping activities are more desirable than other alternatives when individuals use active modes.

\subsection{Interpretation of the estimated coefficients of model $B$}

The estimated values of parameters in the converse choice situation for activity and duration of nonmandatory activities have an inverse relationship and this indicates that individuals aged between 5 and 18 are more likely to choose recreational activities with a short duration. Older people prefer shopping activities rather than other activities in a short duration, and the probability to choose a personal activity in a long duration is higher than other alternatives. Men tend to spend more time doing recreational activities. Choosing a short-duration activity and assigning it to the accompanying alternative have the highest probability for administrative and educational jobs. On the contrary, the retired or homemakers choose the long duration for accompanying trips, which can be attributed to their longer free time than others. The allocation of medium duration to personal activities for retired individuals and homemakers is more likely than others. Besides, people with administrative jobs are less likely to choose the accompanying and recreational alternatives in this duration. Less educated individuals prefer to allocate more time to non-mandatory activities and among all non-mandatory purposes, they prefer shopping activities. However, higher educated people are more likely to devote less time to shopping and recreational activities. The probability of choosing the medium duration for doing recreational activities is 
Table 5. Results of the copula-based model.

\begin{tabular}{|c|c|c|c|c|c|}
\hline Model & \multicolumn{4}{|c|}{ MNL } & \multirow{2}{*}{$\frac{\text { OL }}{\text { Activity time }}$} \\
\hline Variable & Shopping & Personal & Recreational & Convey & \\
\hline Copula dependence parameter & $-1.022(-2.115)$ & $1.850(4.270)$ & $1.380(3.608)$ & $-3.418(-9.106)$ & - \\
\hline Constants & - & $-0.734(-2.677)$ & $-1.495(-7.355)$ & $-2.128(-10.316)$ & $0.947(5.858)$ \\
\hline \multicolumn{6}{|l|}{ Age } \\
\hline Age 5-18 & $-0.470(-3.201)$ & - & - & $-1.285(-4.285)$ & $-0.429(-4.739)$ \\
\hline Age $19-30$ & $-0.261(-2.381)$ & - & - & $-1.44(-7.733)$ & $0.210(3.576)$ \\
\hline Age $31-41$ & - & $0.355(2.966)$ & $-0.179(-1.716)$ & - & - \\
\hline Age $>41$ & - & $0.305(2.830)$ & - & $-0.475(-4.349)$ & - \\
\hline Sex & - & $0.124(1.587)$ & $0.462(4.520)$ & - & $0.251(4.020)$ \\
\hline \multicolumn{6}{|l|}{ Job } \\
\hline ADM. Job & $-0.362(-2.630)$ & $-0.295(-2.338)$ & - & - & $-0.765(-8.322)$ \\
\hline SERV. Job & $-0.930(-3.878)$ & - & - & $0.231(1.805)$ & $-0.908(-10.773)$ \\
\hline EDU. Job & - & - & $1.093(7.560)$ & - & - \\
\hline Other & - & - & $0.682(5.500)$ & - & - \\
\hline DL & - & $-0.116(-1.537)$ & $-0.152(-1.560)$ & $0.573(4.279)$ & $-0.127(-2.089)$ \\
\hline \multicolumn{6}{|l|}{ Education } \\
\hline LOW. EDU & $0.812(5.335)$ & - & $0.366(4.206)$ & - & $0.831(8.716)$ \\
\hline MED. EDU & $0.447(3.162)$ & - & - & - & $0.453(5.142)$ \\
\hline HIGH. EDU & - & $-0.311(-2.307)$ & - & $-0.440(-2.634)$ & - \\
\hline HHSZ & - & $0.138(2.604)$ & - & - & $-0.106(-2.274)$ \\
\hline $\mathrm{CO}$ & - & $-0.205(-3.980)$ & $0.118(1.858)$ & - & $-0.064(-1.577)$ \\
\hline \multicolumn{6}{|l|}{ Mode } \\
\hline Private & $-2.037(-14.293)$ & $-0.939(-7.052)$ & $-1.883(-11.876)$ & - & - \\
\hline Taxi & $-0.696(-8.780)$ & - & $-0.561(-5.973)$ & - & - \\
\hline Bus & - & $0.457(5.238)$ & - & - & $0.249(3.594)$ \\
\hline Motor & $-0.531(-2.720)$ & - & - & - & - \\
\hline Active & - & - & - & $0.804(5.617)$ & $-0.923(-15.792)$ \\
\hline Threshold 1 & - & - & - & - & $0.064(0.763)$ \\
\hline Threshold 2 & - & - & - & - & $1.758(21.829)$ \\
\hline$\rho_{c}^{2}$ & & & 0.107 & & \\
\hline$\rho^{2}$ & & & 0.134 & & \\
\hline
\end{tabular}


Table 6. Results of model A.

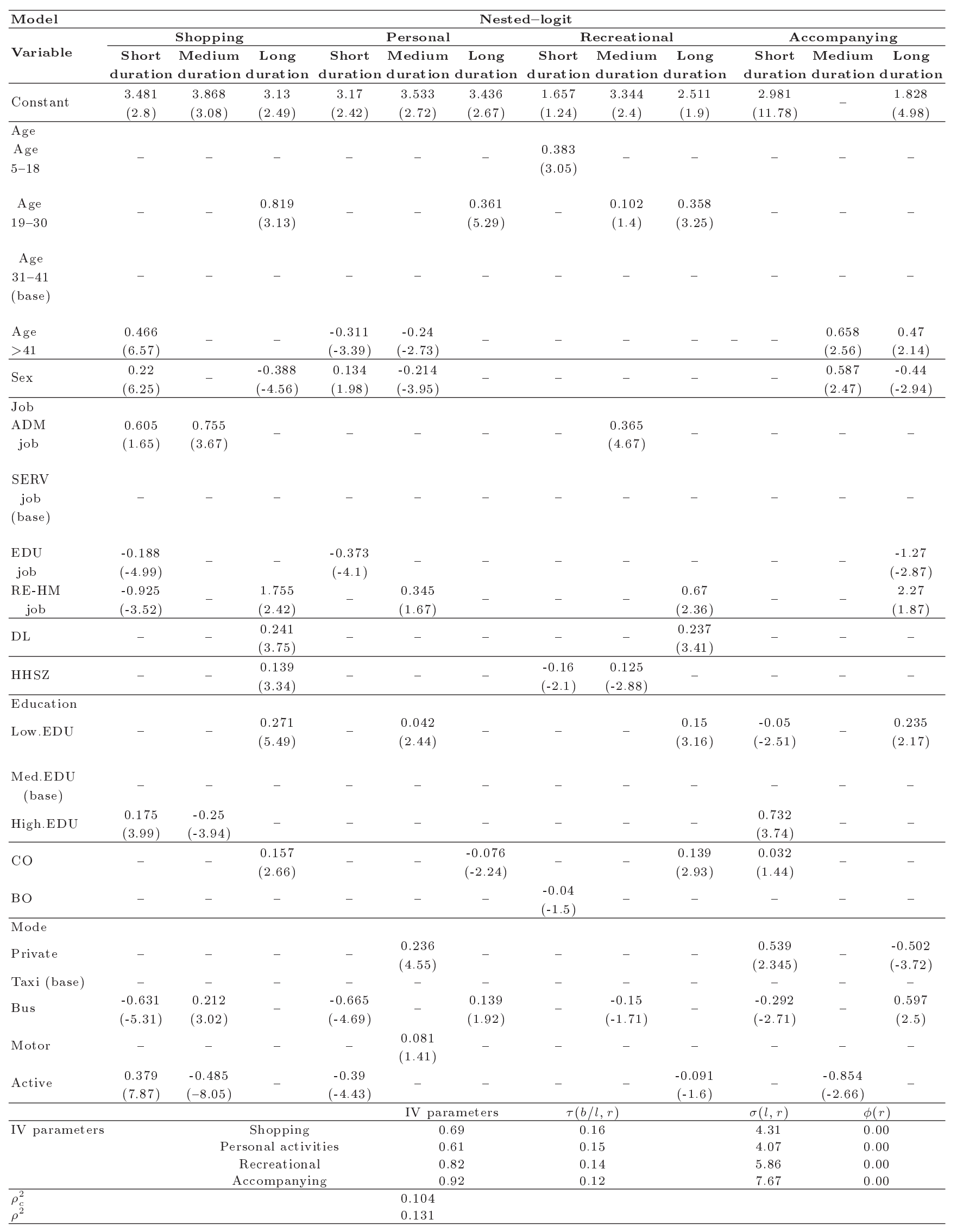




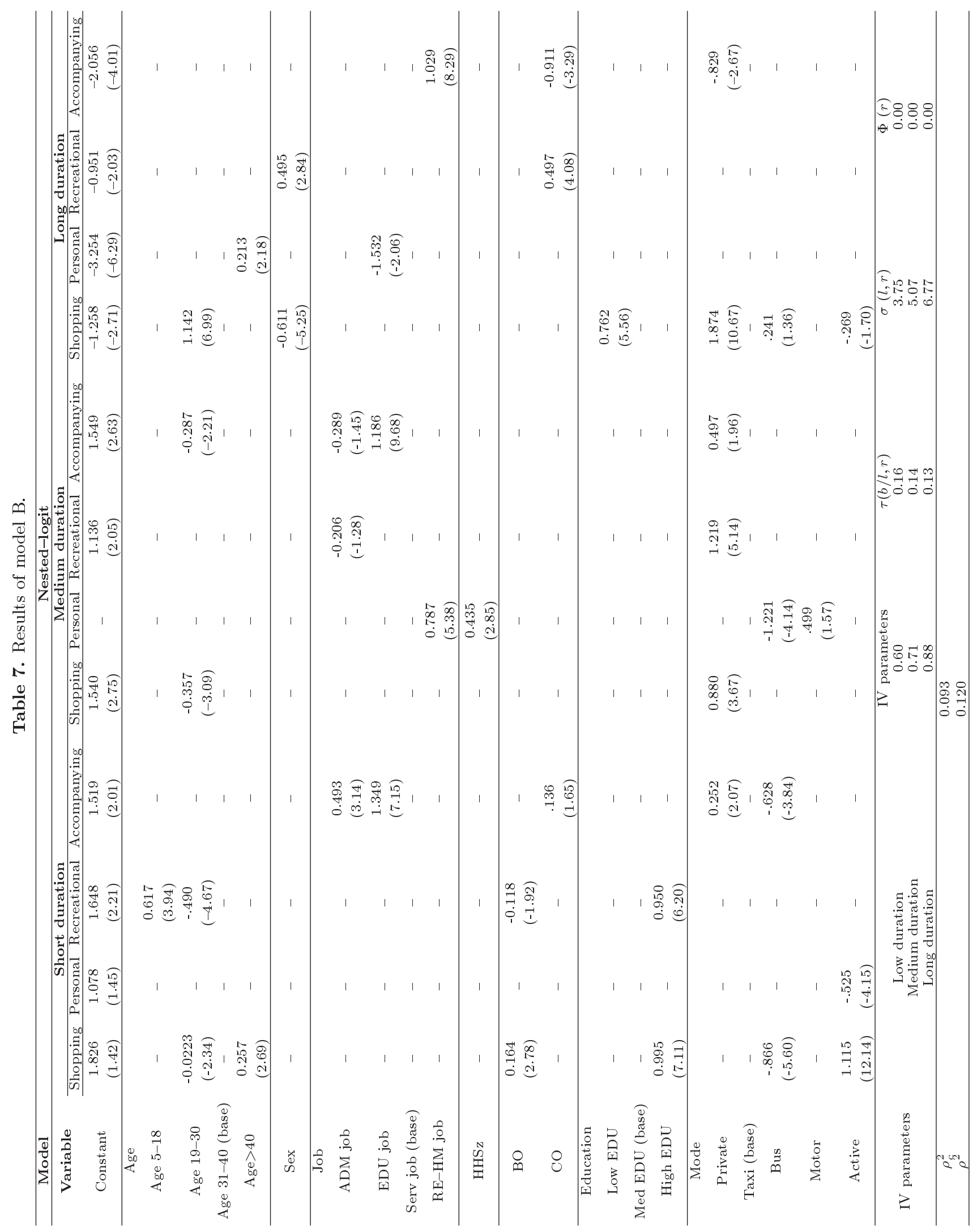


more than other activities with an increase in the number of individuals in a household.

More bicycle ownership leads to the allocation of a shorter duration to recreational activities, and the probability of allocation of short duration to shopping activities decreases. An increase in car ownership leads to a decrease in the probability of choosing longduration accompanying activities. Also, it causes an increase in the probability of choosing long-duration recreational purposes. The coefficients obtained for mode indicate that private car is the appropriate choice for short-duration accompanying activities as well as for all of the non-mandatory activities except personal ones with a medium duration. The probability of choosing this vehicle decreases for long-duration accompanying activities. The bus has the most likelihood of allocating a long duration to shopping activities and the probability that a person assigns a medium duration to personal activities by bus and chooses the short duration for shopping and accompanying activities decreases. By opting for the motorcycle mode, an individual often chooses medium-duration personal trips. If the person chooses the active trip mode, the probability of allocation of long duration to shopping and short duration to personal activities will decrease, while the probability of allocation of short duration to shopping purposes will increase.

\subsection{Comparison of the models}

In addition to the copula-based model, two nested-logit models are also calibrated in this paper in which the activity choice is prioritized over duration choice, and vice versa. The nested-logit model can capture the interaction between these decisions, though it assumes a hierarchical structure between them. To find which decision to assign to the upper level of the hierarchical structure, two nested-logit models with different upper and lower levels are calibrated. The copula-based model does not consider any hierarchical structure and models these decisions jointly. The comparison of the results of the three models highlights two main differences in variable effects. First, the effects of variables on the choice process differ across the two models, only in magnitude. Second, some variables are statistically significant in the nested-logit models (BO in both nested-logit models), while they are statistically insignificant in the copula-based model. Table 8 displays the agreement among the data of the three models.

In addition to the apparent improvement in loglikelihood measures of the copula-based model over the nested-logit models, the models can be evaluated using $\rho_{c}^{2}$ and $\rho_{0}^{2}$. Comparison of the rho-squared values indicates that by modeling these decisions through the joint structure without any hierarchy, an improvement in log-likelihood can be expected. Additionally, nestedlogit with the activity choice at the upper level and activity duration at the lower level has a better structure than another nested-logit model with different levels. In other words, the choice of activity is of higher priority to activity duration.

\subsection{Summary of the findings}

The findings and new results of this study are summarized below:

- Unobserved factors of activity choice and duration choice models were correlated because of estimating high statistical copula dependence parameters;

- The existence of some mutually observed factors confirmed the systematic interaction between these decisions;

- As the nested-logit model had a hierarchical structure, it ignored the mutual interaction between these decisions;

- In terms of $\rho_{0}^{2}, \rho_{c}^{2}$, and $L L(\beta)$, a joint copula-based model outperformed the nested logit models;

- Independent variables related to age, gender, job, having a driving license, educational level, and chosen trip mode of traveller and household size and

Table 8. The fitness of models.

\begin{tabular}{lcccccc}
\hline \multicolumn{1}{c}{ Model } & $\begin{array}{r}\text { Number of } \\
\text { observation }\end{array}$ & $\boldsymbol{L L}(\boldsymbol{\beta})$ & $\boldsymbol{L L}(\boldsymbol{c})$ & $\boldsymbol{L L}(\mathbf{0})$ & $\boldsymbol{\rho}_{\boldsymbol{c}}^{\mathbf{2}}$ & $\boldsymbol{\rho}_{\mathbf{0}}^{\mathbf{2}}$ \\
\hline Joint copula-based & 6536 & -14065 & -15758 & -16241 & 0.107 & 0.134 \\
Nested-logit, & & & & & & \\
$\begin{array}{l}\text { upper level activity choice, } \\
\text { lower level activity duration }\end{array}$ & 6536 & -14129 & -15758 & -16241 & 0.104 & 0.131 \\
& & & & & & \\
$\begin{array}{l}\text { Nested-logit, } \\
\text { upper level activity duration, } \\
\text { lower level activity choice }\end{array}$ & 6536 & -14299 & -15758 & -16241 & 0.093 & 0.120 \\
\hline
\end{tabular}


car ownership of families were influential in activity and duration choice for both joint copula-based and nested logit models. Coefficients of these variables were estimated significantly and logically.

\section{Conclusion}

The present paper aimed to contribute to the existing literature on the choice of non-mandatory activity types and duration. Understanding the choice of nonmandatory activity duration is a very complex task; the diversity and flexibility of such activities are a challenge for modeling travel behavior. In this study, nested-logit and copula-based models were developed to explore the travel behavior, providing an empirical analysis followed by a discussion about the variety of types and duration of non-mandatory activities. The analysis was conducted based on discrete choice models, a copula-based model, and a set of nestedlogit models using the data obtained from Qazvin-Iran in 2012. The models also account for travel-related and socio-demographic variables. It was assumed that travel mode choice came before the choice of activity and duration. Findings highlighted the significant role of socio-demographic characteristics (gender, job, education, car ownership, driver license, and household size) and travel-related (trip mode) factors as the important estimators in the selection of non-mandatory activities and their duration. Furthermore, if decisions about non-mandatory activities and their durations are modeled in the joint structure without any hierarchy, an improvement in log-likelihood and goodness of fit can be expected. Also, nested-logit with the activity choice at the upper level and activity duration at the lower level had a better structure than another nestedlogit model with different levels.

Ultimately, a salient direction for future research would be the consideration of a weekend day to compare and contrast the results. Simultaneous attention to probabilities relating to weekdays and weekends, activity purposes, and duration can be applied to transport planning and land use such as the enhancement of different facilities for spending leisure time.

These models can be used in two ways: to predict future travel demands of society and to describe the relationship between endogenous and exogenous variables.

\section{Abbreviations}

$\begin{array}{ll}\text { MDC } & \text { Multiple Discrete Continues } \\ \text { HAPP } & \text { Household Activity Pattern Problem } \\ \text { GEV } & \text { Generalized Extreme Value } \\ \text { FGM } & \text { Farlie-Gumbel-Morgenster }\end{array}$

\section{References}

1. Bowman, J.L. and Ben-Akiva, M.E. "Activity-based disaggregate travel demand model system with activity schedules", Transportation Research Part a: Policy and Practice, 35(1), pp. 1-28 (2001). https://doi.org/ 10.1016/S0965-8564(99)00043-9

2. Ettema, D., Bastin, F., Polak, J., et al. "Modelling the joint choice of activity timing and duration", Transportation Research Part A: Policy and Practice, 41(9), pp. 827-841 (2007). https://doi.org/10.1016/j. tra.2007.03.001

3. Amirgholy, M. and Gonzales, E.J. "Analytical equilibrium of bicriterion choices with heterogeneous user preferences: application to the morning commute problem", Transportmetrica B: Transport Dynamics, 5(4), pp. 450-482 (2017). https://doi.org/10.1080/ 21680566.2017.1279087.

4. Shabanpour, R., Golshani, N., Auld, J., et al. "Dynamics of activity time-of-day choice", Transportation Research Record, $\mathbf{2 6 6 5 ( 1 ) , ~ p p . ~ 5 1 - 5 9 ~ ( 2 0 1 7 ) . ~}$ https://doi.org/10.3141\%2F 2665-06

5. Bhat, C.R. "A multiple discrete-continuous extreme value model: formulation and application to discretionary time-use decisions", Transportation Research Part B: Methodological, 39(8), pp. 679-707 (2005). https://doi.org/10.1016/j.trb.2004.08.003

6. Bhat, C.R. and Gossen, R. "A mixed multinomial logit model analysis of weekend recreational episode type choice", Transportation Research Part B: Methodological, 38(9), pp. 767-787 (2004). https://doi.org/10.1016/j.trb.2003.10.003

7. Meloni, I., Guala, L., and Loddo, A. "Time allocation to discretionary in-home, out-of-home activities and to trips", Transportation, 31(1), pp. 69-96 (2004). https://doi.org/10.1023/B:PORT.0000007228. 44861.ae

8. Ding, C., Mishra, S., Lin, Y., et al. "Cross-nested joint model of travel mode and departure time choice for urban commuting trips: Case study in MarylandWashington, DC Region", Journal of Urban Planning and Development, 141(4), p. 04014036 (2015). https:// doi.org/10.1061/(ASCE)UP.1943-5444. 0000238

9. Yang, L., Zheng, G., and Zhu, X. "Cross-nested logit model for the joint choice of residential location, travel mode, and departure time", Habitat International, 38, pp. 157-166 (2013). https:// doi.org/10.1016/j.habitatint.2012.06.002

10. Huang, A. and Levinson, D. "Axis of travel: Modeling non-work destination choice with GPS data", Transportation Research Part C: Emerging Technologies, 58, pp. 208-223 (2015). https://doi.org/10.1016/j.trc. 2015.03.022

11. Newman, J.P. and Bernardin, V.L. "Hierarchical ordering of nests in a joint mode and destination choice model", Transportation, 37(4), pp. 677-688 (2010). https://doi.org/10.1007/s11116-010-9277-x 
12. Born, K., Yasmin, S., You, D., et al. "Joint model of weekend discretionary activity participation and episode duration", Transportation Research Record, 2413(1), pp. 34-44 (2014). https://doi.org/10.3141\%2F2413-04

13. Ettema, D.F. and Timmermans, H.J. Activity-Based Approaches to Travel Analysis. Pergamon (1997).

14. Ettema, D. and Timmermans, H. "Modeling departure time choice in the context of activity scheduling behavior", Transportation Research Record, 1831(1), pp. 39-46 (2003). https://doi.org/10.3141\%2F1831-05

15. Axhausen, K.W. "Social networks and travel: Some hypotheses", Social Dimensions of Sustainable Transport: Transatlantic Perspectives, pp. 90-108 (2005).

16. Farber, S. and Páez, A. "My car, my friends, and me: a preliminary analysis of automobility and social activity participation", Journal of Transport Geography, 17(3), pp. 216-225 (2009). https://doi.org/10. 1016/j.jtrangeo.2008.07.008

17. van den Berg, P., Arentze, T., and Timmermans, H. "A latent class accelerated hazard model of social activity duration", Transportation Research Part A: Policy and Practice, 46(1), pp. 12-21 (2012). https://doi.org/10.1016/j.tra.2011.09.015

18. Bhat, C.R. "A hazard-based duration model of shopping activity with nonparametric baseline specification and nonparametric control for unobserved heterogeneity", Transportation Research Part B: Methodological, 30(3), pp. 189-207 (1996). https://doi.org/10. 1016/0191-2615(95)00029-1

19. Ettema, D., Borgers, A.L.O.Y.S., and Timmermans, H.A.R.R.Y. "Competing risk hazard model of activity choice, timing, sequencing, and duration", Transportation Research Record, 1493, p. 101 (1995).

20. Polak, J., Jones, P., Vythoulkas, P., et al. "Travellers'choice of time to travel under road pricing", The 21st PTRC Summer Annual Meeting, 13-16 September, University of Manchester Institute of Science and Technology. TSU Ref (766) (1993).

21. Bhat, C.R. and Steed, J.L. "A continuous-time model of departure time choice for urban shopping trips", Transportation Research Part B: Methodological, 36(3), pp. 207-224 (2002). https://doi.org/10. 1016/S0191-2615(00)00047-3

22. Habib, K.M.N., Day, N., and Miller, E.J. "An investigation of commuting trip timing and mode choice in the Greater Toronto Area: Application of a joint discrete-continuous model", Transportation Research Part A: Policy and Practice, 43(7), pp. 639-653 (2009). https://doi.org/10.1016/j.tra.2009.05.001

23. Habib, K.M.N. "Modeling commuting mode choice jointly with work start time and work duration", Transportation Research Part A: Policy and Practice, 46(1), pp. 33-47 (2012). https://doi.org/10.1016/ j.tra.2011.09.012

24. Popkowski Leszczyc, P.T. and Timmermans, H.J. "Unconditional and conditional competing risk models of activity duration and activity sequencing decisions: An empirical comparison", Journal of Geographical Systems, 4(2), pp. 157-170 (2002). https://doi.org/10.3390/ijgi8120560

25. Chen, C. and McKnight, C.E. "Does the built environment make a difference? Additional evidence from the daily activity and travel behavior of homemakers living in New York City and suburbs", Journal of Transport Geography, 15(5), pp. 380-395 (2007). https://doi.org/10.1016/j.jtrangeo.2006.11.010

26. Kapur, A. and Bhat, C.R. 'Modeling adults' weekend day-time use by activity purpose and accompaniment arrangement", Transportation Research Record, 2021(1), pp. 18-27 (2007). https://doi.org/10. $3141 \% 2 \mathrm{~F} 2021-03$

27. Xu, Z., Kang, J.E., and Chen, R. "A random utility based estimation framework for the household activity pattern problem", Transportation Research Procedia, 23, pp. 809-826 (2017). https://doi.org/10. 1016/j.trpro.2017.05.045

28. Frei, C., Mahmassani, H.S., and Frei, A. "Making time count: Traveler activity engagement on urban transit", Transportation Research Part A: Policy and Practice, 76, pp. 58-70 (2015). https://doi.org/10.1016/ j.tra.2014.12.007

29. Bhat, C.R. and Sen, S. "Household vehicle type holdings and usage: an application of the multiple discrete-continuous extreme value (MDCEV) model", Transportation Research Part B: Methodological, 40(1), pp. 35-53 (2006). https://doi.org/10.1016/ j.trb.2005.01.003

30. Pawlak, J., Polak, J.W., and Sivakumar, A. "A framework for joint modelling of activity choice, duration, and productivity while travelling", Transportation Research Part B: Methodological, 106, pp. 153172 (2017). https://doi.org/10.1016/j.trb.2017.10.010

31. Ermagun, A., Rashidi, T.H., Arian, A., and Samimi, A. "Mode choice and escort decisions in school trips: Application of a copula-based model", TRB 93rd Annual Meeting Compendium of Papers (No. 14-2560) (2014).

32. Rasaizadi, A. and Kermanshah, M. "Mode choice and number of non-work stops during the commute: Application of a copula-based joint model", Scientia Iranica, 25(3), pp. 1039-1047 (2018). https://dx.doi.org/10.24200/sci.2017.4194

33. Bhat, C.R. and Eluru, N. "A copula-based approach to accommodate residential self-selection effects in travel behavior modeling", Transportation Research Part B: Methodological, 43(7), pp. 749-765 (2009). https://doi.org/10.1016/j.trb.2009.02.001

34. Adjei, N.K., Brand, T., and Zeeb, H. "Gender inequality in self-reported health among the elderly in contemporary welfare countries: a cross-country analysis of time use activities, socioeconomic positions and family characteristics", PloS, 12(9), pp. 1-24 (2017). https://doi.org/10.1371/journal.pone.0184676 
35. Liu, X.D., Pan, F., Cai, W.L., et al. "Correlation and risk measurement modeling: A Markovswitching mixed Clayton copula approach", Reliability Engineering \& System Safety, 197, 106808 (2020). https://doi.org/10.1016/j.ress.2020.106808

36. Pan, Y., Ou, S., Zhang, L., et al. "Modeling risks in dependent systems: A copula-Bayesian approach", Reliability Engineering \& System Safety, 188, pp. 416431 (2019). https://doi.org/10.1016/j.ress.2019.03.048

37. Seyedabrishami, S., Rasaizadi, A., Rayaprolu, H.S., et al. "Car ownership: A joint model for number of cars and fuel types", Transportation Research Procedia, 41, pp. 572-576 (2019). https://doi.org/10.1016/j. trpro.2019.09.101

38. Train, K.E. Discrete Choice Methods with Simulation, Cambridge university press (2009).

39. Iran, S.C.O. 2012. (http://www.amar.org.ir in Persian).

40. Rasaizadi, A. and Askari, M. "Effect of family structure on urban areas modal split by using the life cycle concept", Int. J. Hum. Capital Urban Manage, 5(2), pp. $165-174$ (2020).

41. Ewing, R., Schroeer, W., and Greene, W. "School location and student travel analysis of factors affecting mode choice", Transportation Research Record, 1895(1), pp. 55-63 (2004). https://doi.org/10. 3141\%2F1895-08

\section{Biographies}

Fereshteh Jafari Shahdani received her BS degree in Civil Engineering from University of Kurdistan, Kurdistan, Iran in 2015 and her MS in Transportation Planning and Traffic Engineering from Tarbiat
Modares University, Tehran, Iran in 2018, with a thesis entitled "Applying MDCEV model for destination choice in urban arbitrary trips". Currently, she is a PhD fellow within the SAFEWAY project at the University of Minho, Braga, Portugal. Her research work focuses on assessing the indirect costs of flooding as a traffic disruption on urban mobility. Her areas of interest integrate the transport modelling and transport behavior including all modes of transport and transportation network management.

Arash Rasaizadi obtained his BS degree in Civil Engineering from Shahid Bahonar University of Kerman, Kerman, Iran in 2013; he also received his MS degree in Transportation Engineering from Sharif University of Technology (SUT), Tehran, Iran in 2015. He is currently a $\mathrm{PhD}$ candidate in the Transportation Engineering in Tarbiat Modares University (TMU), Tehran, Iran. His research interests include forecast and management of urban travel demand, short-term prediction of traffic parameters, and big data analysis.

Seyedehsan Seyedabrishami received his BS, MS, and $\mathrm{PhD}$ degrees in Transportation Engineering from Sharif University of Technology (SUT) in 2004, 2006, 2011, respectively. He is now an Assistant Professor at the Civil and Environmental Engineering Department at Tarbiat Modares University (TMU) in Tehran. He has recently received a Georg Forster Fellowship as an experienced researcher from the Alexander von Humboldt Foundation in Germany. Since June 2017, he is a Visiting Professor at the Research Group on modeling spatial mobility at the Technical University of Munich (TUM). 